\title{
An Analysis of Finite-Memory Random Linear Coding on Packet Streams
}

\author{
Desmond S. Lun*, Payam Pakzad ${ }^{\dagger}$, Christina Fragouli ${ }^{\dagger}$, Muriel Médard*, and Ralf Koetter ${ }^{\ddagger}$ \\ *Laboratory for Information and Decision Systems \\ Massachusetts Institute of Technology, Cambridge, MA 02139, USA \\ E-mail: \{dslun, medard $\} @$ mit.edu \\ ${ }^{\dagger}$ Laboratoire d'algorithmique \\ Ecole Polytechnique Fédérale de Lausanne, CH-1015 Lausanne, Switzerland \\ E-mail: \{payam.pakzad, christina.fragouli\}@epfl.ch \\ $\ddagger$ Coordinated Science Laboratory \\ University of Illinois at Urbana-Champaign, Urbana, IL 61801, USA \\ E-mail: koetter@uiuc.edu
}

\begin{abstract}
We consider the following packet coding scheme: The coding node has a fixed, finite memory in which it stores packets formed from an incoming packet stream, and it sends packets formed from random linear combinations of its memory contents. We analyze the scheme in two settings: as a selfcontained component in a network providing reliability on a single link, and as a component employed at intermediate nodes in a block-coded end-to-end connection. We believe that the scheme is a good alternative to automatic repeat request (ARQ) when feedback is too slow, too unreliable, or too difficult to implement.
\end{abstract}

\section{INTRODUCTION}

It was recently shown [1], [2], [3] that, if every node in a network sends out packets formed from random linear combinations of previously received packets, then capacity is achieved between any source and any sink (or any source and any set of sinks) in a lossy packet network. There are two main drawbacks of such a scheme: The first is memory usage. Since it is assumed that nodes store all previously received packets, the memory usage of intermediate nodes grows with $K$, the size of the coding block at the source. This requirement that all previously received packets be stored can be relaxed, but the memory usage of intermediate nodes must nevertheless grow with $K$. The second drawback is computational complexity. Sinks decode by performing Gaussian elimination on the packets they receive to invert the linear coding operations, which, on average, requires $O\left(K^{3}\right)$ operations. Thus, decoding is a polynomial-time procedure, but, given the existence of erasure-correcting codes with linear decoding complexity [4], [5], we would expect that lower decoding complexity is perhaps possible.

In this paper, we consider a simple way of improving at least one, and possibly both, of these two drawbacks. It comes, however, with the cost that we may not necessarily achieve capacity. We consider fixing the memory of intermediate nodes

This work was supported by the National Science Foundation under grant nos. CCR-0093349, CCR-0325496, and CCR-0325673, by Hewlett-Packard under grant no. 008542-008, and by the Air Force Office of Scientific Research under grant no. F49620-01-1-0365. at some constant with respect to $K$. Thus, from the point of view of the encoder at a particular intermediate node, a stream of packets of indeterminate length arrives, and the encoder functions much like a convolutional encoder. There is, however, one very important difference between the coding we consider and convolutional coding on packets [6]: Our coding scheme is rateless in the sense that the coded packet stream can maintain any ratio that we desire to the rate of the message packet stream and can be freely adjusted in real-time. By contrast, packet-level convolutional coding can adapts its rate only by puncturing the code, which allows variation only within a limited range. This ability to freely adapt the rate of the code allows the scheme to vary its erasure-correcting capability to meet time-varying conditions, which are common in packet networks.

We consider the use of our coding scheme in two settings: first, as a self-contained component in a network providing reliability on a single link; and, second, as a component employed at intermediate nodes in a block-coded end-to-end connection. We shall see that, by explicitly recognizing that the scheme is being employed as a component at intermediate nodes in a block-coded end-to-end connection, better performance can be achieved than if we did not.

We begin by describing the coding scheme in the following section. We consider its use as a self-contained component in Section III and as a component in a block-coded end-to-end connection in Section IV.

\section{CODE DESIGN}

The design of the coding scheme is simple. We assume that packets are vectors of length $L$ over the finite field $\mathbb{F}_{q}$. Hence, if the packet length is $b$ bits, then $L=\left\lceil b / \log _{2} q\right\rceil$. The encoder has a memory capable of storing $m$ packets, and it uses this memory in one of two ways:

1) as a shift register: arriving packets are stored in memory and, if the memory is already full, the oldest packet in the memory is discarded; or 
2) as an accumulator: arriving packets are multiplied by a random vector chosen uniformly over $\mathbb{F}_{q}^{m}$, and the product is added to the $m$ memory slots.

To form coded packets, the encoder simply takes a random linear combination (in $\mathbb{F}_{q}$ ) of its memory contents, with the vector of coefficients of the combination drawn uniformly from $\mathbb{F}_{q}^{m} \backslash\{0\}$. This can be done as often or as seldom as we wish; hence the ratelessness of the code-the output of coded packets does not need to be synchronized to the arrival of packets in any way and, in particular, does not need to be related to it according to some fixed coding rate.

The coding scheme we propose results essentially from taking the coding scheme described in [1], [2], [3] for a single intermediate node and limiting the encoder's memory to a size that is fixed with respect to the input. Related random linear coding schemes are described in [7], [8] for the application of multicast over lossless networks, in [9] for data dissemination, and in [10] for data storage.

\section{USE AS A SELF-CONTAINED COMPONENT}

When used as a self-contained component, the encoder takes an incoming stream of packets, $u_{1}, u_{2}, \ldots$, and forms a coded stream of packets that is placed on its lossy outgoing link and decoded on reception. The decoder, we assume, knows the linear transformations that the packets it receives are of the message packets $u_{1}, u_{2}, \ldots$. This information can be communicated to the decoder by a variety of means, which include placing it into the header of each packet (which is certainly viable when the memory is used as a shift registerthe overhead is $m \log _{2} q$ bits plus that of a sequence number), and initializing the random number generators at the encoder and decoder with the same seed.

The task of decoding, then, equates to matrix inversion in $\mathbb{F}_{q}$, which can be done straightforwardly by applying Gaussian elimination to each packet as it is received. This procedure produces an approximately-steady stream of decoded packets with an expected delay that is constant in the length of the input stream. Moreover, if the memory is used as a shift register, then the complexity of this decoding procedure is also constant with the length of the input stream and, on average, is $O\left(m^{2}\right)$ per packet.

\section{A. Model}

We discretize the time axis into epochs that correspond to the transmission of an outgoing packet. Thus, in each epoch, an outgoing packet is transmitted, which may be lost, and one or more incoming packets are received. If transmission is to be reliable, then the average number of incoming packets received in each epoch must be at most one.

We adopt the following simple model of incoming packet arrivals and outgoing packet losses, with the understanding that generalizations are certainly possible. We assume that, in an epoch, a single packet arrives independently with probability $r$ and no packets arrive otherwise, and the transmitted outgoing packet is lost independently with probability $\varepsilon$ and is received otherwise. This model is appropriate when losses and arrivals are steady — and not bursty.

\section{B. Analysis}

The following analysis is in the limit of $q \rightarrow \infty$, i.e. the limit of infinite field size. We later discuss how the analysis may be adapted for finite $q$, and, in Section III-C, we quantify by simulation the difference between the performance in the case of finite $q$ and that of infinite $q$ in some particular instances.

We begin by considering the difference between the number of packets received by the encoder and the number of packets transmitted and not lost. This quantity, we see, evolves according to the infinite-state Markov chain shown in Figure 1, where $\alpha=r \varepsilon, \beta=(1-r)(1-\varepsilon)$, and $\gamma=r(1-\varepsilon)+(1-r) \varepsilon$.

At epoch 0 , the memory of the encoder is empty and we are in state 0 . We continue to remain in state 0 in subsequent epochs at least until the first packet $u_{1}$ arrives. Suppose the next outgoing packet is not lost. Then we remain in state 0 , and the decoder receives a packet that is a random linear combination of $u_{1}$, i.e. a random scalar multiple of $u_{1}$, and, since $q$ is infinitely large by assumption, this scalar multiple is non-zero with probability 1 ; so the decoder can recover $u_{1}$ from the packet that it receives.

Now suppose the next outgoing packet is lost; so we move to state 1 . If an outgoing packet is transmitted and not lost before the next packet arrives, then we again receive a random scalar multiple of $u_{1}$ and return to state 0 . So suppose $u_{2}$ arrives. Then, the next outgoing packet is a random linear combination of $u_{1}$ and $u_{2}$. Suppose further that this packet is received by the decoder, so we are again in state 1. This packet, currently, is more or less useless to the decoder; it represents a mixture between $u_{1}$ and $u_{2}$ and does not allow us to determine either. Nevertheless, it gives the decoder some information that it did not previously know, namely, that $u_{1}$ and $u_{2}$ lie in a particular linear subspace of $\mathbb{F}_{q}^{2}$. Any subsequent packet received by the decoder, then, also gives the decoder information that it did not previously know, provided that the linear combination that it is formed from is linearly independent of the one already received. We call such an informative packet innovative.

What we see is that, provided that packets arrive only in states $0,1, \ldots, m-1$, then every packet that is transmitted from a non-zero state is innovative at the decoder if it is not lost, and, at every return to state 0 , the decoder is able to recover one or more packets. If a packet arrives in state $m$, then losses start to occur. Information in the encoder's memory is overwritten or corrupted, and will never be recovered. The current contents of the encoder's memory, however, can still be recovered and, from the point of view of recovering these contents, the coding system behaves as though we were in state $m$. Hence, to analyze the performance of the coding scheme, we modify the Markov chain shown in Figure 1 to that in Figure 2. Let $x_{t}$ be the state of this Markov chain at time $t$. We can interpret $x_{t}$ as the number of innovative packets the encoder has to send at time $t$.

We now proceed to derive some quantities that are useful for designing the parameters of the coding scheme. We begin with 


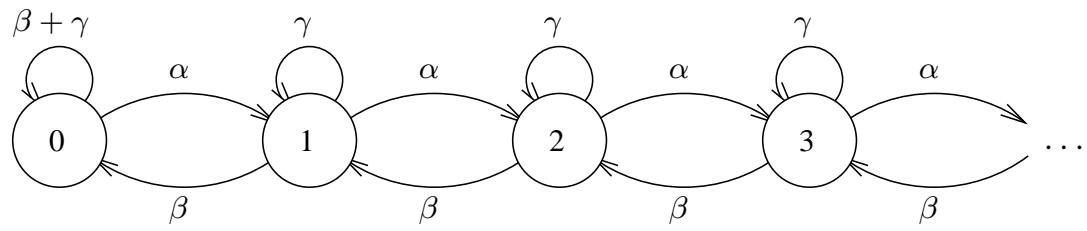

Fig. 1. Markov chain modeling the evolution of the difference between the number of packets received by the encoder and the number of packets transmitted and not lost.

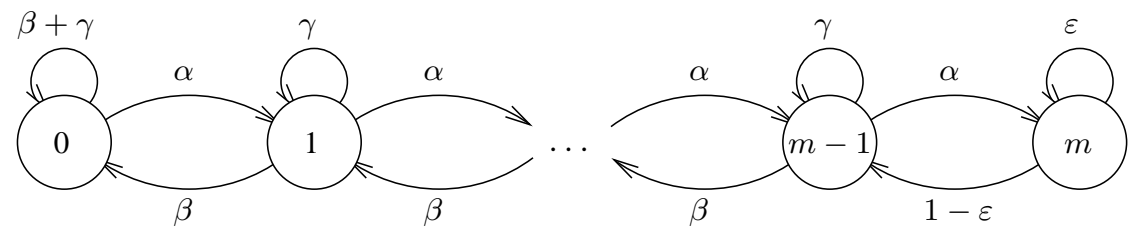

Fig. 2. Markov chain modeling the behavior of the coding scheme in the limit of $q \rightarrow \infty$.

the steady-state probabilities $\pi_{i}:=\lim _{t \rightarrow \infty} \operatorname{Pr}\left(x_{t}=i\right)$. Since $\left\{x_{t}\right\}$ is a birth-death process, its steady-state probabilities are readily obtained. We obtain

$$
\pi_{i}=\frac{\rho^{i}(1-\rho)}{1-\sigma \rho^{m}}
$$

for $i=0,1, \ldots, m-1$, and

$$
\pi_{m}=\frac{\varepsilon \sigma \rho^{m-1}(1-\rho)}{1-\sigma \rho^{m}}
$$

where $\rho:=\alpha / \beta=r \varepsilon /(1-r)(1-\varepsilon)$ and $\sigma:=r /(1-\varepsilon)$. We assume $\rho<1$, which is equivalent to $r<1-\varepsilon$, for, if not, the capacity of the outgoing link is exceeded, and we cannot hope for the coding scheme to be effective.

We now derive the probability of packet loss, $p_{l}$. Evaluating $p_{l}$ is not straightforward because, since coded packets depend on each other, the loss of a packet owing to the encoder exceeding its memory is usually accompanied by other packet losses. We derive an upper bound on the probability of loss.

A packet is successfully recovered by the decoder if the ensuing path taken in the Markov chain in Figure 2 returns to state 0 without a packet arrival occurring in state $m$. Let $q_{i}$ be the probability that a path, originating in state $i$, reaches state 0 without a packet arrival occurring in state $m$. Our problem is very similar to a random walk, or ruin, problem (see, for example, [11, Chapter XIV]). We obtain

$$
q_{i}=\frac{1-\sigma \rho^{m-i}}{1-\sigma \rho^{m}}
$$

for $i=0,1, \ldots, m$.

Now, after the coding scheme has been running for some time, a random arriving packet finds the scheme in state $i$ with probability $\pi_{i}$ and, with probability $1-\varepsilon$, the scheme returns to state $i$ after the next packet transmission or, with probability $\varepsilon$, it moves to state $i+1$. Hence

$$
\begin{aligned}
& 1-p_{l} \geq \sum_{i=0}^{m-1}\left\{(1-\varepsilon) q_{i}+\varepsilon q_{i+1}\right\} \pi_{i} \\
&=\frac{1}{\left(1-\sigma \rho^{m}\right)^{2}}\left\{1-\rho^{m}-(1-2 \varepsilon) m \sigma \rho^{m}\right. \\
&\left.\quad-\varepsilon m \sigma \rho^{m-1}+(1-\varepsilon) m \sigma \rho^{m+1}\right\} .
\end{aligned}
$$

From which we obtain

$$
\begin{gathered}
p_{l} \leq \frac{\rho^{m-1}}{\left(1-\sigma \rho^{m}\right)^{2}}\{\varepsilon m \sigma+(1-2 \sigma+m \sigma-2 \varepsilon m \sigma) \rho \\
\left.-(1-\varepsilon) m \sigma \rho^{2}+\sigma^{2} \rho^{m+1}\right\} .
\end{gathered}
$$

We have thus far looked at the limit of $q \rightarrow \infty$, while, in reality, $q$ must be finite. There are two effects of having finite $q$ : The first is that, while the encoder may have innovative information to send to the decoder (i.e. $x_{t}>0$ ), it fails to do so because the linear combination it chooses is not linearly independent of the combinations already received by the decoder. For analysis, we can consider such non-innovative packets to be equivalent to erasures, and we find that the effective erasure rate is $\varepsilon\left(1-q^{-x_{t}}\right)$. The Markov chain in Figure 2 can certainly be modified to account for this effective erasure rate, but doing so makes analysis much more tedious.

The second of the effects is that, when a new packet arrives, it may not increase the level of innovation at the encoder. When the memory is used as a shift register, this event arises because a packet is overwritten before it has participated as a linear factor in any successfully received packets, i.e. all successfully received packets have had a coefficient of zero for that packet. When the memory is used as an accumulator, this event arises because the random vector chosen to multiply the new packet results in the dimension of the encoder's memory not being increased. The event of the level of innovation not being increased by a new packet can be quite disastrous, because it is effectively equivalent to the encoder exceeding its memory. Fortunately, the event seems rare; in the accumulator 

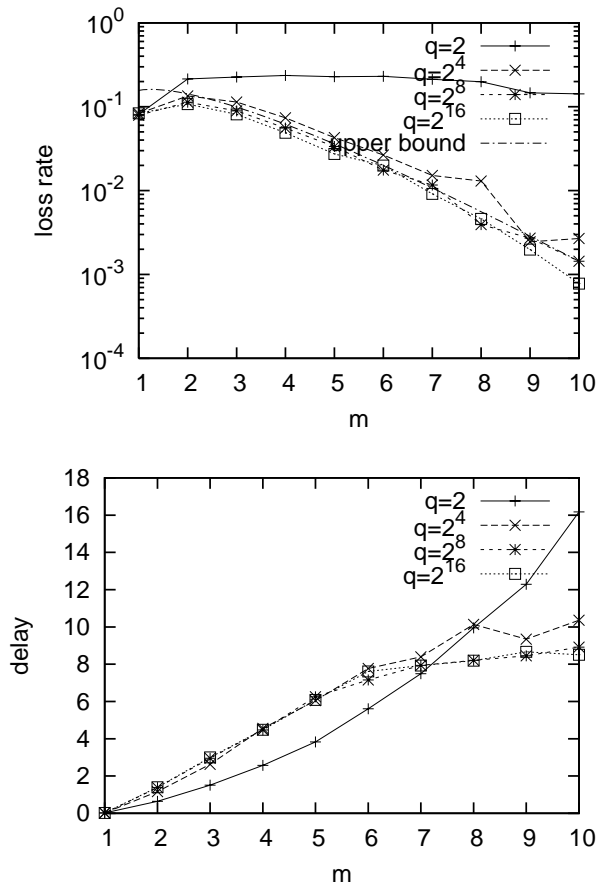

Fig. 3. Average loss (top) and delay (bottom) for 200,000 packets as a function of memory size $m$ with $r=0.8, \varepsilon=0.1$, and various coding field sizes $q$. The upper bound on the probability of loss for $q \rightarrow \infty$ is also drawn

case, we can quantify the probability of the event exactly as $1-q^{x_{t}-m}$.

\section{Simulation results}

We chose $\varepsilon=0.1$ and simulated the performance of our coding scheme for 200,000 packets with various choices of the parameters $r, q$, and $m$ (see Figures 3 and 4). We decoded using Gaussian elimination on packets as they were received and used the encoder's memory as a shift register to keep decoding complexity constant with the length of the packet stream. Delay was evaluated as the number of epochs between a packet's arrival at the encoder and it being decoded, neglecting transmission delay. We see that a field size $q \geq 2^{8}$ (perhaps even $q \geq 2^{4}$ ) is adequate for attaining loss rates close to the upper bound for infinite field size.

\section{USE IN A BLOCK-CODED END-TO-END CONNECTION}

When our coding scheme is used as a self-contained component, packets are sometimes lost because the decoder receives linear combinations that, although innovative, are not decodable. For example, suppose the decoder receives $u_{1}+u_{2}$, but is neither able to recover $u_{1}$ nor $u_{2}$ from other packets. This packet, $u_{1}+u_{2}$, definitely gives the decoder some information, but, without either $u_{1}$ or $u_{2}$, the packet must be discarded. This would not be the case, however, if $u_{1}$ and $u_{2}$ were themselves coded packets - a trivial example, assuming that we are not coding over $\mathbb{F}_{2}$, is if $u_{1}=u_{2}=w_{1}$, where $w_{1}$ is a message packet for an outer code.

In such a case, we can use the encoder described in Section II, but we should not attempt to recover the stream fed
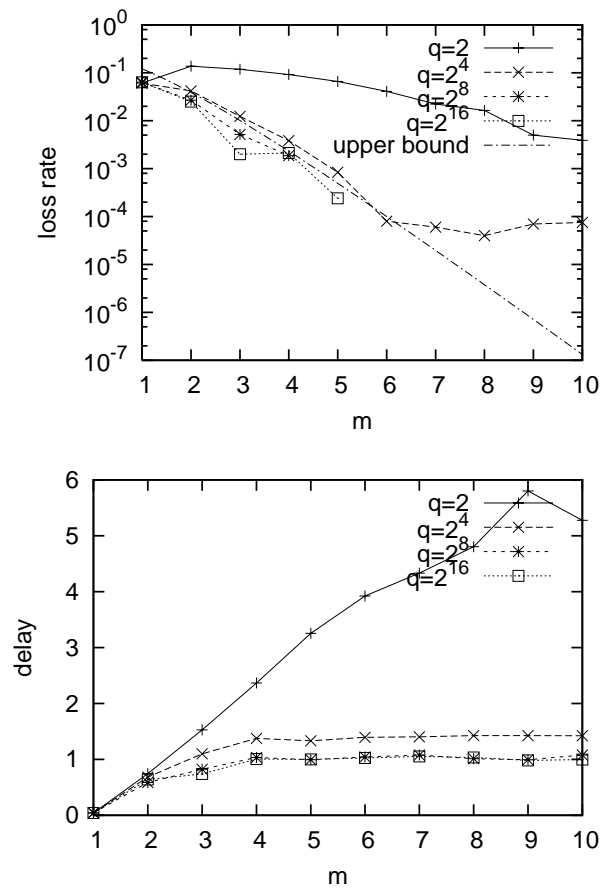

Fig. 4. Average loss (top) and delay (bottom) for 200,000 packets as a function of memory size $m$ with $r=0.6, \varepsilon=0.1$, and various coding field sizes $q$. The upper bound on the probability of loss for $q \rightarrow \infty$ is also drawn. Average losses of zero are not shown.

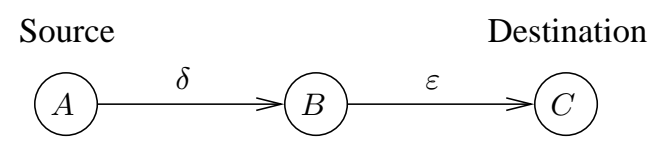

Fig. 5. A two-link tandem network.

to the encoder with the procedure detailed in Section III, or rather, if we do, then the decoder should preserve any packets that are linearly independent after Gaussian elimination, not only those that are copies of packets in the encoder's input stream.

In this section, we consider using our coding scheme in the simplest network with end-to-end coding and intermediate node coding: a two-link tandem network with coding at the intermediate node (see Figure 5). This simple two-link tandem network that we analyze serves as a basis for longer tandem networks and more general network topologies.

The source $A$ has $K$ message packets $w_{1}, w_{2}, \ldots, w_{K}$ that it encodes with an erasure code into $N$ packets $u_{1}, u_{2}, \ldots, u_{N}$. These packets are sent over link $A B$ with loss rate $\delta$ to the intermediate node $B$. Node $B$ uses our coding scheme to encode the packets that it receives, and sends coded packets over link $B C$ with loss rate $\varepsilon$ to the destination $C$. Node $C$, now, cares only about receiving $K$ packets that are linearlyindependent transformations of $u_{1}, u_{2}, \ldots, u_{N}$ because, from these, it has an invertible linear transformation of the original message packets $w_{1}, w_{2}, \ldots, w_{K}$. Recovering $w_{1}, w_{2}, \ldots, w_{K}$ at $C$ is done by Gaussian elimination. 


\section{A. Model}

We again adopt a discrete-time model. This model, which is identical to that used in [12], supposes that packets are transmitted on both links $A B$ and $B C$ at each epoch and that packets transmitted on $A B$ and $B C$ are lost independently with probability $\delta$ and $\varepsilon$, respectively. Although actual networks may not have transmissions that are synchronized in this way, the synchronicity assumption may be relaxed to an extent by accounting for differences in the packet injection rates on links $A B$ and $B C$ using the loss rates $\delta$ and $\varepsilon$.

\section{B. Analysis}

We again conduct our analysis in the limit of infinite field size. The considerations for finite field size are the same as those mentioned in Section III-B.

Let $x_{t}$ denote the number of innovative packets (relative to $u_{1}, u_{2}, \ldots, u_{N}$ ) node $B$ has to send at time $t$, and let $y_{t}$ denote the number of innovative packets received by node $C$ at time $t$. By the arguments of Section III-B, the following principles govern the evolution of $x_{t}$ and $y_{t}$ over time:

- As long as $x_{t}<m$, i.e. the memory is not fully innovative, node $B$ increases the innovation contents of its memory by 1 upon successful reception of a packet over link $A B$.

- As long as $x_{t}>0$, i.e. the memory is not completely redundant, the output of $B$ is innovative, so $y_{t}$ will increase by 1 provided that transmission over $B C$ is successful.

Let $\alpha:=(1-\delta) \varepsilon, \beta:=\delta(1-\varepsilon)$, and $\lambda:=(1-\delta)(1-\varepsilon)$. Then the evolution of $x_{t}$ and $y_{t}$ is modeled by the Markov chain shown in Figure 6, where the horizontal coordinate of a state indicates $x_{t}$, and the vertical coordinate corresponds to the variable $y_{t}$.

We see that $\left\{x_{t}\right\}$ evolves as in Section III-B, so its steadystate probabilities are given by (1) and (2) with $r=1-\delta$. Hence, once the system is sufficiently mixed, the probability that $y_{t}$ increases at time $t$ is given by

$$
\begin{aligned}
\lambda \pi_{0}+(1-\varepsilon) \pi_{1}+\cdots+(1-\varepsilon) \pi_{m} & =(1-\varepsilon)\left(1-\delta \pi_{0}\right) \\
& =(1-\delta)\left(1-\pi_{m}\right) .
\end{aligned}
$$

Therefore the system can operate at rate

$$
R=(1-\delta)\left(1-\pi_{m}\right)
$$

with high probability of success.

Suppose, without loss of generality, that $\delta>\varepsilon$, so $\rho<1$. Let $R^{*}$ be the min-cut capacity, or maximum rate, of the system, which, in this case, is $1-\delta$. Then the relative rate loss with respect to the min-cut rate is

$$
1-\frac{R}{R^{*}}=\pi_{m}
$$

\section{Simulation results}

As discussed before, the analysis of Section IV assumes forming linear combinations over an infinitely large field, resulting in a Markov chain model with transition probabilities

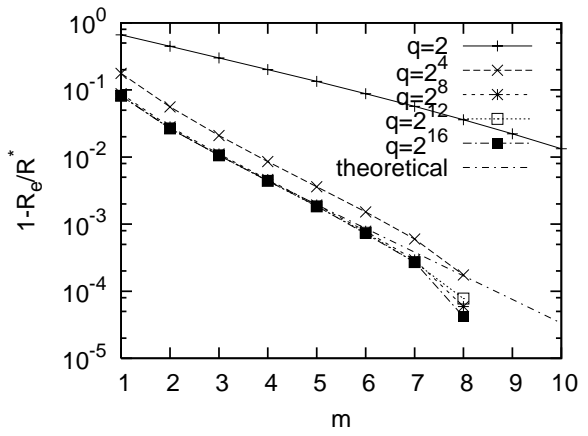

Fig. 7. Relative rate loss with respect to min-cut rate as a function of memory size $m$ for $\delta=0.2, \varepsilon=0.1$, and various coding field sizes $q$.

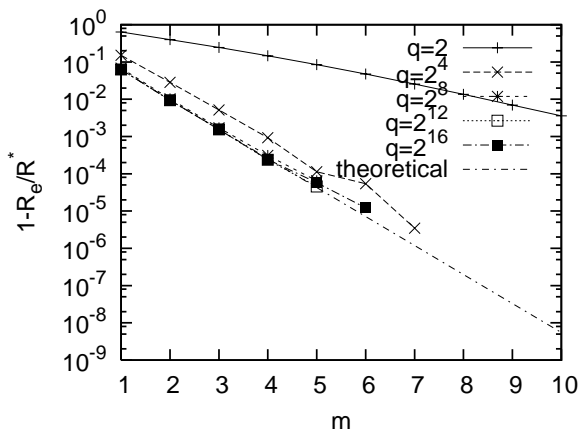

Fig. 8. Relative rate loss with respect to min-cut rate as a function of memory size $m$ for $\delta=0.4, \varepsilon=0.1$, and various coding field sizes $q$.

given in Figure 6. If on the other hand the field size is finite, we can still find new expressions for the transition probabilities, although the complete analysis becomes very complex. In particular, assume that the memory is used as an accumulator, so that the contents of the memory at each time are uniformly random linear combinations, over $\mathbb{F}_{q}$, of the received packets at $B$ by that time. Then, as we have mentioned, if the innovation content of the memory is $x$ and a new packet arrives at $B$, the probability that $B$ can increase the innovation of its memory by 1 is $\left(1-q^{x-m}\right)$, independently from all other past events. Similarly, the probability that the output of $B$ is innovative is $\left(1-q^{-x}\right)$.

To quantify the effect of operations over a finite field, we simulated the evolution of this Markov chain for two combinations of $\delta$ and $\epsilon$ values that were also considered in Section III (see Figures 7 and 8). The effective rate is considered to be $R_{e}:=y_{N} / N$, where $N$ is the number of packet transmissions at $A$, and as before, $y_{N}$ is the number of innovative packets received at $C$ by time $N$. We simulated this process for $N=10^{9}$ packets. For different field sizes, we plot the relative rate loss with respect to the min-cut rate-i.e. $1-R_{e} / R^{*}$-as a function of the memory size. Also plotted is the theoretical result from (5).

By comparing Figures 7 and 8 with Figures 3 and 4, respectively, we see the advantage that comes from explicitly recognizing that the coding scheme is being employed at the 


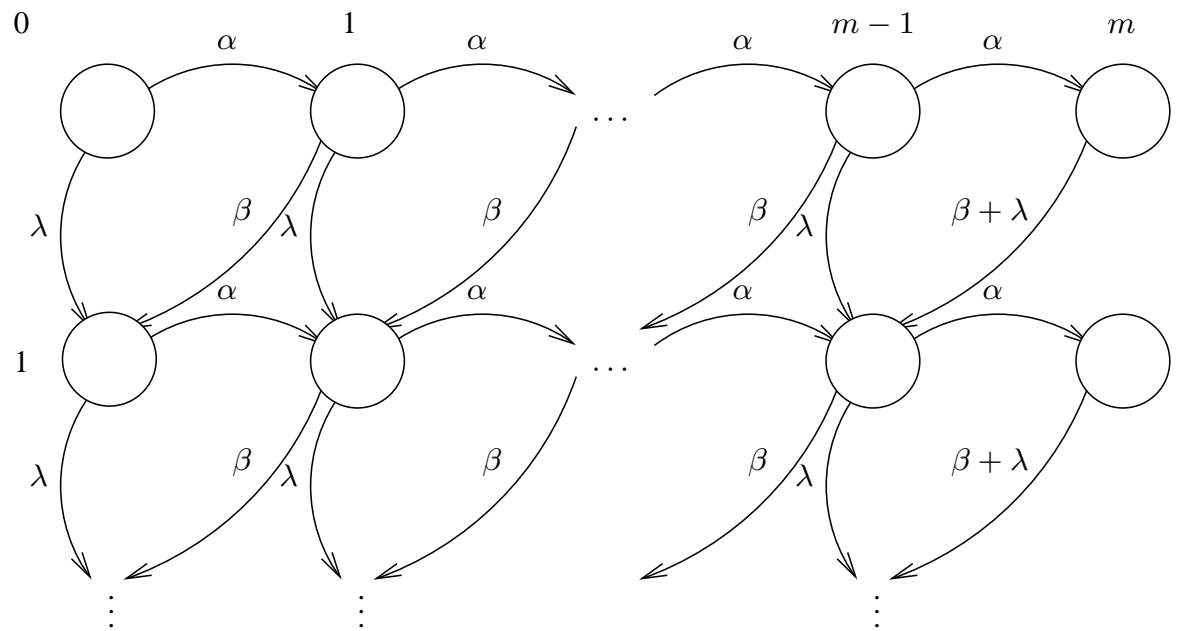

Fig. 6. Markov chain modeling the evolution of $x_{t}$ and $y_{t}$. To simplify the diagram, we do not show self-transitions.

intermediate node of a block-coded end-to-end connection. The loss rate in the latter plots essentially equates to the factor $1-R_{e} / R^{*}$ in the former. Thus, in the limit of infinite $q$, we compare the probability of loss $p_{l}$ upper bounded by equation (3) and the expression for $1-R / R^{*}$ given by equation (5). We note that, in both cases, the decay as $m \rightarrow \infty$ is as $\rho^{m}$. Moreover, it follows from our discussion that $1-R / R^{*}$ must be a lower bound for $p_{l}$, hence $p_{l}$ itself decays as $\rho^{m}$ as $m \rightarrow \infty$.

\section{CONCLUSION}

We have analyzed the performance of finite-memory random linear coding and seen that it shows promise as a means of providing reliability in packet networks. This task is performed in many current packet networks using ARQ, which relies on feedback. But feedback is sometimes either not available or not of high enough quality, and then feedforward coding schemes such as ours become useful. We therefore believe that our coding scheme is a good alternative to ARQ when feedback is too slow, too unreliable, or too difficult to implement. (Indeed, the Markov chain in Figure 2 also describes the behavior of stop-and-wait ARQ with delay-free feedback.)

If some feedback is available, however, it can be used to assist our coding scheme. For example, feedback messages can be sent from the decoder when the state of the coding scheme approaches $m$, which the encoder should respond to either by descreasing the code rate, by increasing $m$, or both. This strategy requires some level of feedback that is less than that required by an ARQ scheme and, indeed, by varying the threshold state above which feedback messages are sent, it is possible to obtain a full trade off of coding with feedback.

Looking more broadly, we see from this and other work that random linear coding, as a general technique, is a promising way of providing reliability and enabling multicast in packet networks and that, while various implementation issues have been discussed, no clear picture guiding the path to a protocol has yet been offered. This picture, we believe, should include the finite-memory considerations of this paper as well as the use of feedback, perhaps even allowing us to view ARQ as a special case.

\section{ACKNOWLEDGMENTS}

The authors would like to thank Amin Shokrollahi for helpful discussions and suggestions.

\section{REFERENCES}

[1] D. S. Lun, M. Médard, R. Koetter, and M. Effros, "On coding for reliable communication over packet networks," submitted to IEEE Trans. Inform. Theory.

[2] — , "Further results on coding for reliable communication over packet networks," in Proc. 2005 IEEE International Symposium on Information Theory (ISIT 2005), Sept. 2005, pp. 1848-1852.

[3] D. S. Lun, M. Médard, and M. Effros, "On coding for reliable communication over packet networks," in Proc. 42nd Annual Allerton Conference on Communication, Control, and Computing, Sept.-Oct. 2004, invited paper.

[4] P. Maymounkov, “Online codes," NYU, Technical Report TR2002-833, Nov. 2002.

[5] A. Shokrollahi, "Raptor codes," Jan. 2004, preprint.

[6] M. Arai, S. Fukumoto, and K. Iwasaki, "Reliability analysis of a convolutional-code-based packet level FEC under limited buffer size," IEICE Trans. Fundamentals, vol. E88-A, no. 4, pp. 1047-1054, Apr. 2005.

[7] P. A. Chou, Y. Wu, and K. Jain, "Practical network coding," in Proc. 41st Annual Allerton Conference on Communication, Control, and Computing, Oct. 2003.

[8] T. Ho, M. Médard, R. Koetter, D. R. Karger, M. Effros, J. Shi, and B. Leong, "Toward a random operation of networks," submitted to IEEE Trans. Inform. Theory.

[9] S. Deb and M. Médard, "Algebraic gossip: A network coding approach to optimal multiple rumor mongering," submitted to IEEE Trans. Inform. Theory.

[10] S. Acedański, S. Deb, M. Médard, and R. Koetter, "How good is random linear coding based distributed networked storage?" in Proc. WINMEE, RAWNET and NETCOD 2005 Workshops, Apr. 2005.

[11] W. Feller, An Introduction to Probability Theory and its Applications, 3rd ed. New York, NY: John Wiley \& Sons, 1968, vol. 1.

[12] P. Pakzad, C. Fragouli, and A. Shokrollahi, "Coding schemes for line networks," in Proc. 2005 IEEE International Symposium on Information Theory (ISIT 2005), Sept. 2005. 